\title{
Realization of Signal Acquisition System in Seismoelectric Logging
}

\author{
Pan ZHOU a and Xiang-Yang SUN \\ University of Electronic Science and Technology of China, Chengdu 610054, China
}

\begin{abstract}
With the prosperous development of electronic science and technology, electrical logging technology has been widely used in the process of oil exploitation. Seismoelectric logging is one of the many electrical logging methods, which has some unique advantages compared with other logging methods. At present, there are a lot of theoretical research on the seismoelectric logging at home and abroad, but due to some engineering problems has not been solved, practical instrument is not yet come into being. The acquisition of seismoelectric signal is one of the most difficult problems in the practical application. In this paper, a set of seismoelectric signal acquisition system based on SPI bus is designed to solve this problem. Experiment show that the system basically meets the demand of signal acquisition in seismoelectric logging
\end{abstract}

\section{Introduction}

There are many kinds of oil logging methods, such as electric logging, acoustic logging, radioactive logging and so on[1]. Generally speaking, all kinds of logging methods can directly or indirectly reflect the characteristics of some aspects of the underground rock stratum, but it is necessary to use a variety of logging methods in order to fully evaluate the underground rock stratum. Seismoelectric logging is a kind of electrical logging, which based on the coupling mechanism of mechanical energy and electromagnetic energy, can directly detect the properties of formation, such as permeability, conductivity, porosity, and concentration of well liquid ion, with high resolution and excellent probing ability[2]. Compared with other logging methods, seismoelectric logging has its unique advantages and can serve as a useful complement to other logging methods. The research on seismoelectric logging has been started since the 90s of last century, but logging instrument based on this method is still not available. The reason is that there are still some key technical difficulties unsolved in engineering application, one of the major difficulties is the acquisition of underground seismoelectric signals.

Essentially, the seismoelectric signal is a electromagnetic signal, which is generated by the mechanical vibration in the fluid medium. In the process of seismoelectric logging, the logging system needs to coordinate the acquisition process of many measuring points underground.At the same time, a large number of data produced by each measurement point needs to be transmitted to the host computer in real time, which is the biggest difficulty for seismoelectric signal acquisition.

\footnotetext{
${ }^{a}$ Corresponding author: 15994237697@qq.com
} 


\section{Basic process of seismoelectric logging}

The seismoelectric logging system usually uses acoustic wave as the source of vibration, which is easy to produce and also easy to adjust. A complete seismoelectric logging system, as shown in Figure 1, consists of three parts: acoustic emission system, seismoelectric signal acquisition system, host computer. In some cases, the seismoelectric logging device and acoustic logging device are integrated into one system, because the two logging methods have similarities in the principle of implementation, and can be complementary in function.

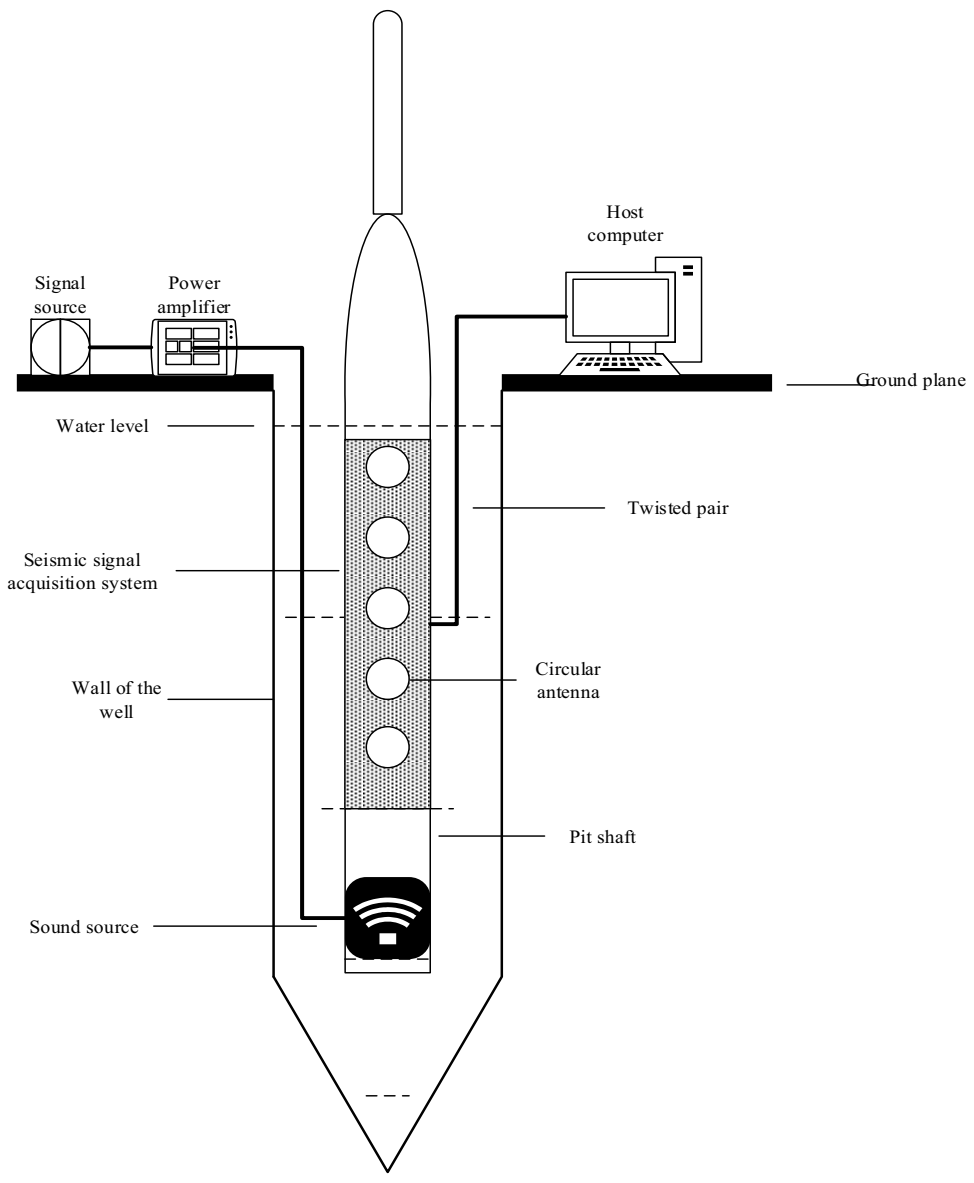

Figure 1. Basic frame of seismoelectric logging system

The acoustic emission system generates a square pulse signal to excite the sound source through the power amplifier,then the sound source generates a strong acoustic wave. Due to the existence of the seismoelectric effect, the acoustic wave will excite the seismoelectric signal in the porous medium when it propagates along the borehole wall.

The seismoelectric logging system is used to collect the electrical signals generated in different depths.Then,it will transfer the measurement data to the host computer immediately. In the host 
computer, the parameters and properties of the the underground rock stratum are evaluated according to the collected data.

\section{Design of seismoelectric signal acquisition system}

In this paper, a SPI bus based seismoelectric signal acquisition system is presented. The system block diagram is shown in Figure 2, which includes two parts: data acquisition board and control board.

A total of eight data acquisition board, placed in the eight cavities of the pit shaft respectively, to collect seismoelectric signal of different depths. The trigger signal is generated by the acoustic emission system, which is a level pulse signal, and used to synchronize the data acquisition process of the eight acquisition board. When an effective trigger signal is sent out, the eight acquisition board starts to collect the seismoelectric signal synchronously. The data acquisition board receives the electrical signal by antennas, and then store the relevant data in storage space. The acquisition board will not send the data to the SPI bus until it dectect an effective control signal in the CS(Control Signal) line.

The control board sequentially selects the data acquisition board through the SPI bus in order to control the data transmission process. After the data acquisition board detects an effective CS signal, the data is transmitted from acqusition board to the control board through the SDI(Signal Data In) line in accordance with the timing of the SPI bus protocol. After receiving all the data of the current acquisition board, the control board will transfer the data to the host computer through the twisted pair cable.Then,control board switch to the next channel through CS line and repeat the data transfer operation above.

In the host computer, the data of seismoelectric signal will be used to evaluate the properties and parameters of underground formation[3].

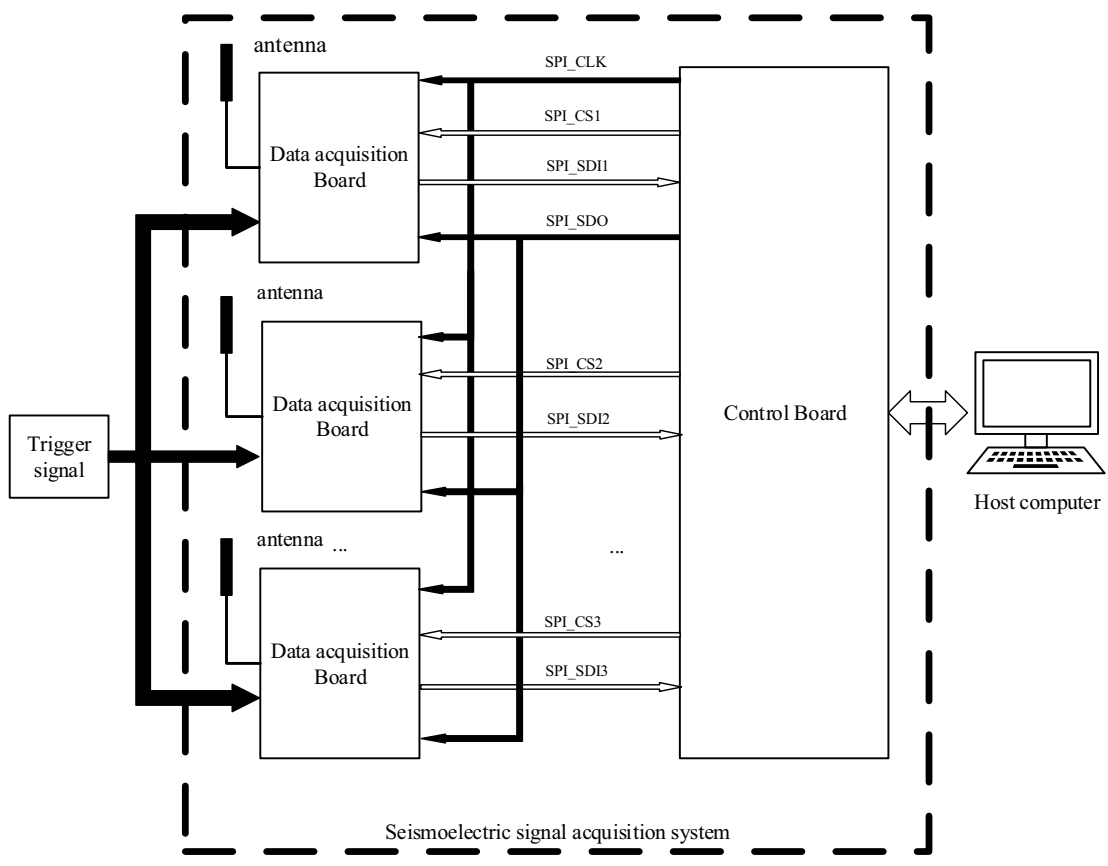

Figure 2. Diagram of seismoelectric signal acquisition system 


\section{Simulative well experiment}

\subsection{Experimental environment}

Build an experimental environment to simulate the underground environment. The experimental facility comprises a water tank, grindstone, pit shaft.

Put the stone into the water tank, and then add enough water to drown the grindstone. Next, add a certain amount of salt to the water to change the ion concentration of the solution so that the seismoelectric signals are more easily detected.

Place the antennas, data acquisition boards, control board and sound source in their right place of the pit shaft respectively, as shown in Figure 3. After taking waterproofing measures, put the shaft into the tank and close to the grindstone.

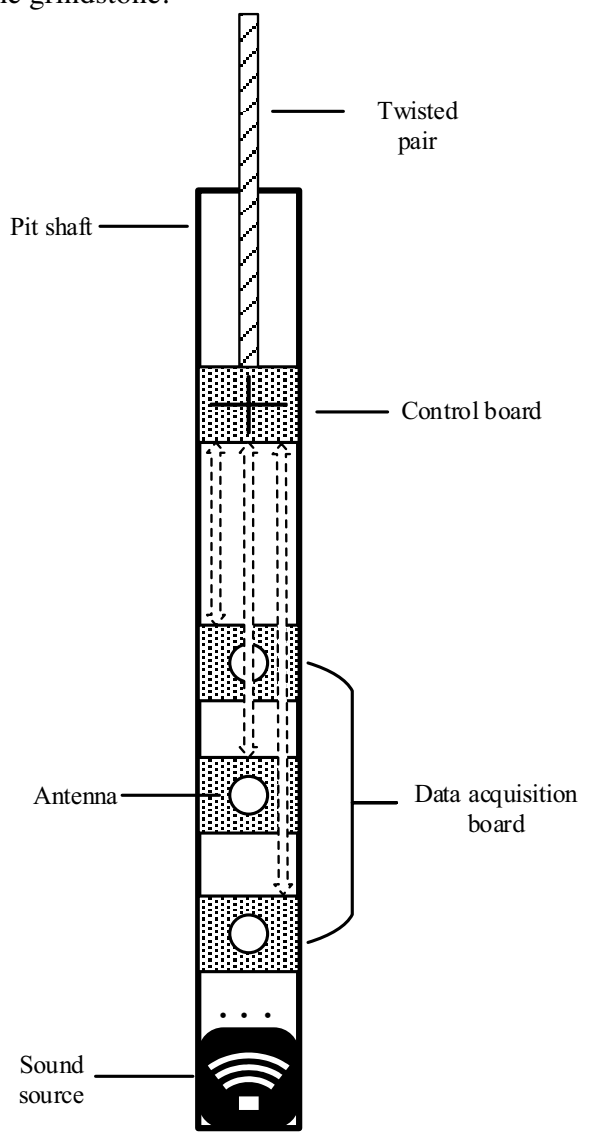

Figure 3. Installation diagram of the seismoelectric signal acquisition system

\subsection{Experimental equipment}

The experimental equipment includes signal source, power amplifier, sound source, oscilloscope, computer and so on. 


\subsection{Experimental principle}

As can be seen from Figure 1, a complete seismoelectric logging system consists of three parts: the acoustic emission system, the seismoelectric signal acquisition system and the host computer.

A square wave pulse signal is generated by the signal source, and then amplified by the power amplifier. Amplified signal then is sent to the sound source,so the sound source will produce a strong sound wave. Because of the seismoelectric effect, acoustic shock will generate electrical signals in the surface of the grindstone. After the seismoelectric signal is received by the seismoelectric signal acquisition system, a series of processing will be carried out, and then related data will be transmitted to the host computer through the control board. At last,the host computer is used to analyze the data and judge the validity of the seismoelectric data.

\subsection{Experimental conditions}

-Excitation type to sound source: square pulse (voltage amplitude $=600 \mathrm{v}$,pulse width $=65 \mu \mathrm{v}$ );

-Antenna type: helical antenna, as shown in Figure 4;

-Spacing of adjacent antennas: $0.15 \mathrm{~m}$;

-Sampling frequency of data acquisition board: $260 \mathrm{KHz}$;

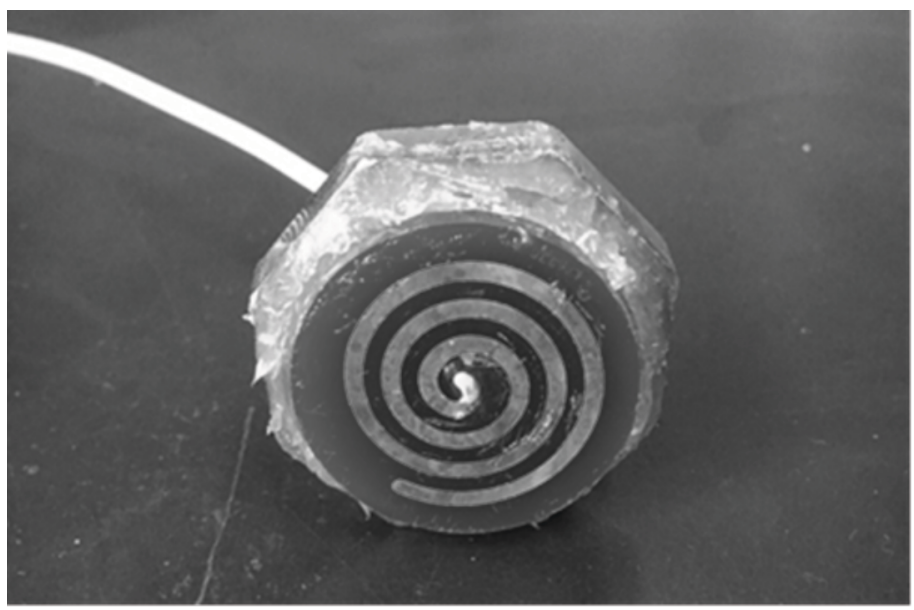

Figure 4. Waterproof helical antenna.

\subsection{Experimental results}

In actual measurement, there is a strong correlation bettwen the data of seven channel,while noise and seismoelectric signal are overlapped in the collected data of the remaining channel.Here we only analysis the seven channel that data has strong correlation.

Using origin to draw the seven channel data, as shown in Figure 5. 


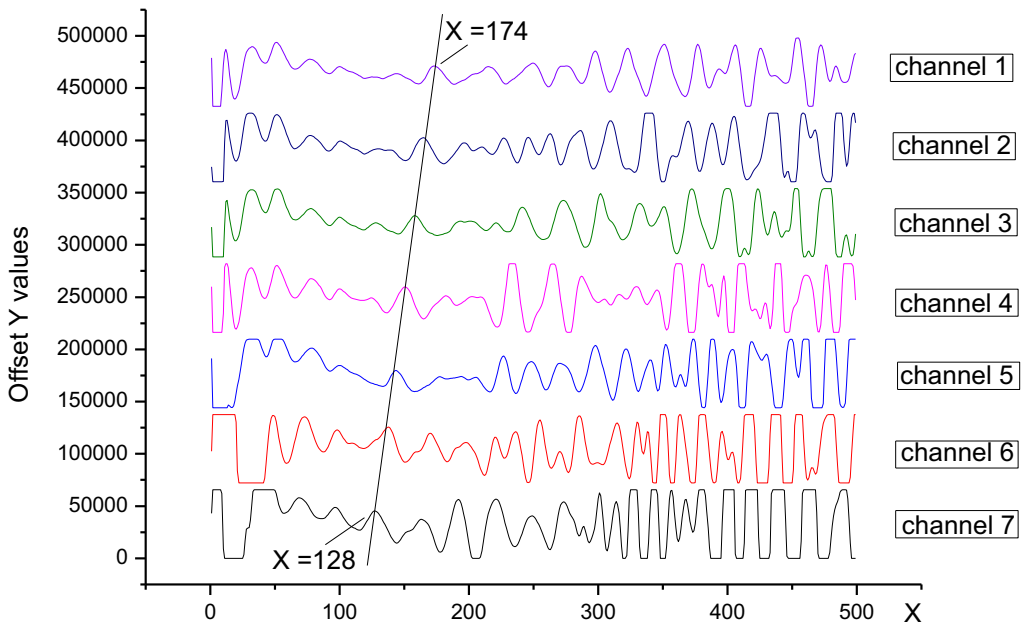

Figure 5. Experimental data waveform.

In Figure 5, the data of the channel 1 to the channel 7 is collected by data acqusition board from up to down respectively.The waveform of each channel not only include the seismoelectric signal, but also the noise. The main source of noise is the power amplifier. The power amplifier produces a very strong excitation, and the frequency of which is very close to the seismoelectric signal, so the electromagnetic wave of excitation will be received together with the seismoelectric signal by data acquisition board.

But we can distinguish the noise and the electrical signal from the waveform above .In Figure 4, the power amplifier is placed on the ground, and the noise signal is transmitted from the ground to underground. The sound source is placed at the bottom of the shaft, and the sound waves propagate along the wall from bottom to top. According to the analysis above, the waveform in front of the slash are mainly the noise, while the waveform behind the slash are mainly the seismoelectric signal.

According to the time interval between the seismoelectric signal waveform of different channel, it is also able to calculate the velocity of acoustic wave propagated along the borehole.

Figure 5 shows that the interval data points between channel 1 and the channel 7 is 46 in the abscissa . Known from 4.4 that the sampling frequency of the data acquisition board is $260 \mathrm{KHz}$, so we can calculate that the time interval between adjacent data points is $3.85 \mu$ s. Therefore, the time that sound spend to propagate from acquisition board 1 to acquisition board 7 is:

$$
3.85 \mu \mathrm{s} * 46=177.1 \mu \mathrm{s}
$$

In addition, the distance between the acquisition board 1 and the acquisition board 7 is:

$$
0.15 m * 6=0.9 m
$$

Therefore, it can be calculated that the velocity of sound wave propagated along the borehole wall:

$$
0.9 m \div 177.1 \mu_{s} \approx 5081 \mathrm{~m} / \mathrm{s}
$$


This is consistent with the velocity of sound wave propagated in the rock $(4500 \mathrm{~m} / \mathrm{s}$ to $7500 \mathrm{~m} / \mathrm{s})$, which confirms that the seismoelectric signal acquired by acquisition board is arose from the acoustic wave[4].

\section{Conclusion}

Seismoelectric logging is a very promising oil logging technology, but the acquisition of seismoelectric signal is one of the most difficult problems in engineer application. This paper designs a set of signal acquisition system that is able to coordinate multiple measurement points to acquire underground seismoelectric signals of different depths underground. Simultaneously, the system can transmit all the data to the host computer in real time through SPI bus. It basically meets the demand of signal acquisition in seismoelectric logging and has certain application value in engineering.

\section{Acknowledgements}

This work was financially supported by the State's Key Project of Research and Development Plan(20 16YFC0303502), and supported by “the Fundamental Research Funds for the Central Universities" (Z YGX2015J019).

\section{References}

1. Segesman F F. Well-logging method[J]. Geophysics, 45,1667-1684(2012)

2. Russell R D, Butler K E, Kepic A W, et al. Seismoelectric exploration[J]. Leading Edge, 16,1611-1615(1997)

3. Zyserman F I, Monachesi L B, Jouniaux L. Dependence of shear wave seismoelectrics on soil textures: a numerical study in the vadose zone[J]. (2017)

4. Wang Shiguang,Sheng Yang,He Hanhan, Effect of petrophysical property on the propagation of ultrasonic in rocks. Physics Experimentation,8,9-13(2014) 